\title{
Automatic processing and analysis of the structural properties of bone tissue
}

\author{
Elena Semenova ${ }^{1, *}$, Nikita Kharin $^{1}$, Pavel Bolshakov ${ }^{1,2}$, Anastasiya Ivanova $^{1}$, and \\ Viktoriya Yaikova ${ }^{1}$ \\ ${ }^{1}$ Kazan Federal University, 18, Kremlevskaya str., Kazan, Russian Federation \\ ${ }^{2}$ Kazan National Research Technical University named after A.N. Tupolev, Kazan, 10, Karl Marx \\ str., Kazan, Russian Federation
}

\begin{abstract}
The volumetric distribution of bone tissue can be analysed in terms of orthotropic medium. In this case, it is important to define the orthotropic directions. Nowadays, computed tomography methods allow getting such information. The method for automation such analysis is presented. Firstly, the threshold of binarization should be calculated. Then the sample should be meshed and each element should be binarized. After that fabric tensor, eigenvalues, eigenvectors and fractional anisotropy can be calculated for each element. Statistical methods were used to analyse the field of the obtained data. Described methods were used on a bone sample. It was shown that for a sample the fabric tensor is constant and the fractional anisotropy is close to zero. That's means that the medium in the sample was isotropic.
\end{abstract}

\section{Introduction}

Modern medical treatment is most often based on biomechanical modeling. This approach improves the quality of treatment. In this case, it is important to know the mechanical parameters of the tissue [1-4]. Obviously, the classical approach from composite mechanics can be used. But it is difficult to carry out verification experiments [5-8]. Therefore, computed tomography methods have become increasingly popular. It has been shown that the mechanical properties of bone tissue correlate with computed tomography data [9-12]. This means that quantitative information about the quality of bone tissue can be obtained at the diagnostic stage. Currently, there are various methods for assessing the orthotropic properties of the medium, such as representative models [13-15], inhomogeneous FE models [16, 17], direct FE models [18] and mixed methods [19-21]. Another problem of such analysis is to obtain the distribution of mechanical properties in the object under study. The actual task in these problems is the automation of such a calculation. The obtained data can be useful in various biomechanical calculations, such as determination of bone defects before [22-25] and after [26-28] surgery.

\footnotetext{
*Corresponding author: elena.semionova2011@rambler.ru
} 


\section{Materials and methods}

The volume distribution of bone tissue can be analyzed in terms of an orthotropic medium. In this case, it is important to define orthotropic directions. The tissue tensor is currently used to estimate orthotropic directions. The Average Intersection Length (MIL) method is used to construct the tissue tensor. The MIL in a material can be calculated as the aspect ratio of the distance, measured along a straight line, between two components of the material [19]. In this case, the fabric tensor can be calculated as the square root of the inverse approximation matrix. Usually the quadratic form $[19,27,29]$ is used to approximate the MIL:

$$
L^{-2}(n)=n \cdot M 4 \cdot n,
$$

where is $\boldsymbol{n}$ the unit vector in the direction of the mean intercept length measurement and length $L(\mathbf{n})$ is the mean intercept length. To estimate the degree of anisotropy, we introduce fractional anisotropy, that can be calculated using the eigenvalues of the fabric tensor:

$$
r=\sqrt{\frac{1}{2}} \cdot \frac{\sqrt{\left(\lambda_{1}-\lambda_{2}\right)^{2}+\left(\lambda_{2}-\lambda_{3}\right)^{2}+\left(\lambda_{3}-\lambda_{1}\right)^{2}}}{\sqrt{\lambda_{1}^{2}+\lambda_{2}^{2}+\lambda_{3}^{2}}} \cdot 100 \%
$$

where $\lambda_{1}-$ is the $1^{\text {st }}$ eigenvalue, $\lambda_{2}-$ is the $2^{\text {nd }}$ eigenvalue, $\lambda_{3}-$ is the $3^{\text {rd }}$ eigenvalue.

In the case of isotropy material (all eigenvalues are equal), the fractional anisotropy is equal to zero.

Previous technique [29] for MIL approximation was generalized for three-dimensional space. The MIL data in a material can be represented as a vector:

$$
x_{i}=\left(x_{i}, y_{i}, z_{i}\right), \mathrm{i}=\overline{1, n} \text {. }
$$

In this case we can present quadratic form in general case as:

$$
f(x)=x \cdot A \cdot x^{T}+x \cdot B+C=0,
$$

where

$$
A=\left(\begin{array}{ccc}
A_{11} & 2 A_{12} & 2 A_{13} \\
2 A_{12} & A_{22} & 2 A_{23} \\
2 A_{13} & 2 A_{23} & A_{33}
\end{array}\right), \mathrm{B}=\left(\begin{array}{l}
B_{1} \\
B_{2} \\
B_{3}
\end{array}\right) .
$$

The problem can be solved by minimization the target function:

$$
J(x)=\sum_{i=1}^{n}(f(x))^{2} \rightarrow \min .
$$

We introduce the vector of unknown parameters of the target function:

$$
\eta=\left(A_{11}, A_{12}, A_{22}, A_{23}, A_{13}, A_{33}, B_{1}, B_{2}, B_{3}, C\right)^{T} .
$$

In this case, the problem of minimization the target function leads to:

$$
\frac{\partial J(x, \eta)}{\partial \eta_{i}}=0(i=\overline{1,10}) \text {. }
$$

This problem is equal to system of linear equations [29, 30]:

$$
S \cdot \eta=b
$$


Here constant $C$ is just a scale factor of our approximation. According to a well-known inverse tensor theorem, quadratic form $A$ is a second rank symmetric tensor.

We used a specific algorithm to analyze the CT data. Generally, the algorithm of the analysis can be described as $[25,29,31,32]$ :

1) calculating the binarization threshold;

2) meshing the sample;

3) binarizing of each element;

4) constructing the MIL and its approximating, and calculating the fabric tensor for each element;

5) calculating eigenvalues, eigenvectors and fractional anisotropy of fabric tensor;

6) analyzing received fields for the object.

Statistical methods were used to analyze the eigenvector field. To exclude fluctuations in the directions of eigenvectors, the most possible direction was determined. Thus, the most possible orthotropic directions can be determined. Then the dependence of orthotropic directions on spatial coordinates was investigated. To understand the behavior of orthotropic directions in a bone sample, the following method was used. The cylindrical coordinate system was constructed with the center at the center of mass of the sample. The center of each element was recalculated in the new coordinate system. In this case, a dataset $U$ can be created:

$$
U=\left(r_{i}, \theta_{i}, \varphi_{i} \mid v a l_{i}\right)
$$

where $r, \theta, \varphi$ - cylindrical coordinates of $i^{\text {th }}$ element, val - data of interest (e.g. fractional anisotropy or eigenvectors).

Singular value decomposition can be used for the correlation matrix of the dataset $U$. The singular values were normalized to their maximum value and a threshold of $10 \%$ was used. The discarded values will be understood as not affecting the data of interest.

\section{Results and discussion}

For numerical problem, CT data of rat's femur diaphysis was used. Micro/nanofocal X-ray inspection system for CT and 2D inspections of Phoenix V / tome I X S240 was used for scanning. The system is equipped with two X-ray tubes: microfocus with a maximum accelerating voltage of $240 \mathrm{kV}$ power of $320 \mathrm{~W}$ and nanofocus with a maximum accelerating voltage of $180 \mathrm{kV}$ power of $15 \mathrm{~W}$. For primary data processing and creating a volume (voxel) model of the sample based on x-ray images, the datos / $x$ reconstruction software was used. The sample fixed in the holder was placed on the rotating table of the $\mathrm{X}$-ray computed tomography chamber at the optimum distance from the X-ray source. The survey was carried out at an accelerating voltage of $90-100 \mathrm{kV}$ and current $140-150 \mathrm{~mA}$, the dimension of a voxel is $6.747 \mu \mathrm{m}$. Mesh is shown on fig. 1b (totally 280 elements).

The binarization threshold was found using the Otsu method. On fig. 2 original data (a), binarized (b) and MIL with approximation (c) is shown. The described method was used for each element of the mesh. Received data was eigenvalues, eigenvectors, and fractional anisotropy. In fig. 3a distribution of eigenvectors is shown, red lines are the $1^{\text {st }}$ eigenvector, green lines are the $2^{\text {nd }}$ eigenvector and blue lines are the $3^{\text {rd }}$ eigenvector. In fig. $3 \mathrm{~b}$ distribution of fractional anisotropy is shown. This distribution can be useful to discover isotropic and anisotropic zones in the object. 


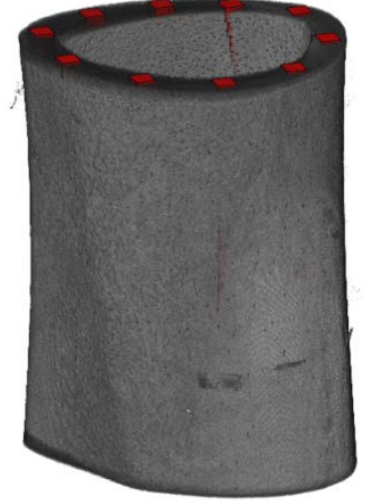

a

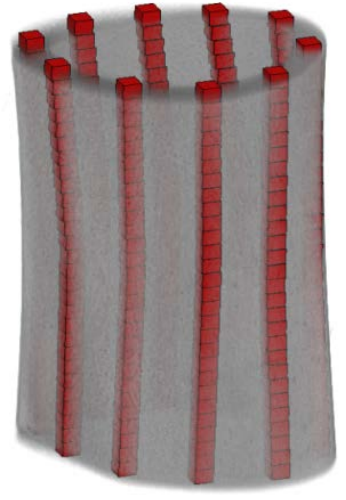

b

Fig. 1. Example of CT data (a) and it's mesh(b).

Distribution of eigenvectors and fractional anisotropy is shown in fig. 3. Using described statistical methods it can be shown that eigenvalues and eigenvector fields are constant. This fact is confirmed by values in the distribution of fractional anisotropy, which are in the range from $1 \%$ to $6 \%$. It means that the medium in the sample is isotropic.

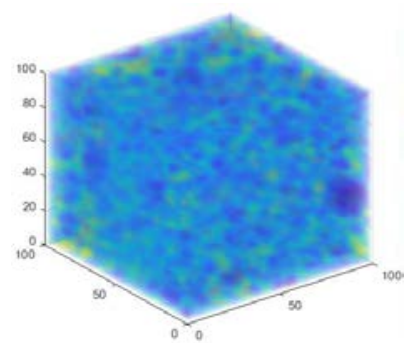

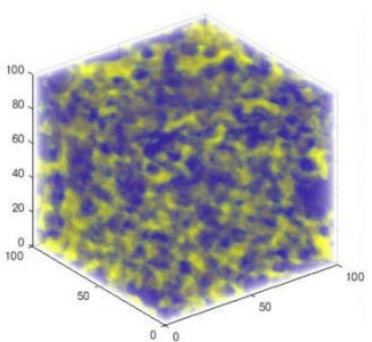

$\mathrm{b}$

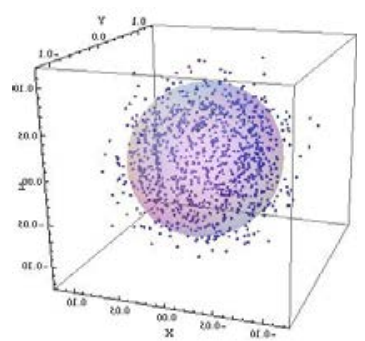

c

Fig. 2. Element: (a) - origin data, (b) - binarized data, (c) - MIL and it's approximation.

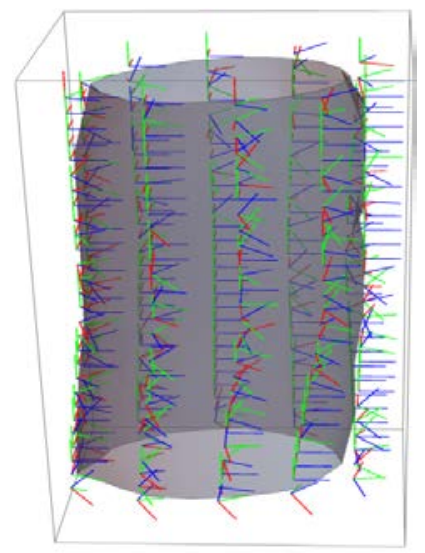

a

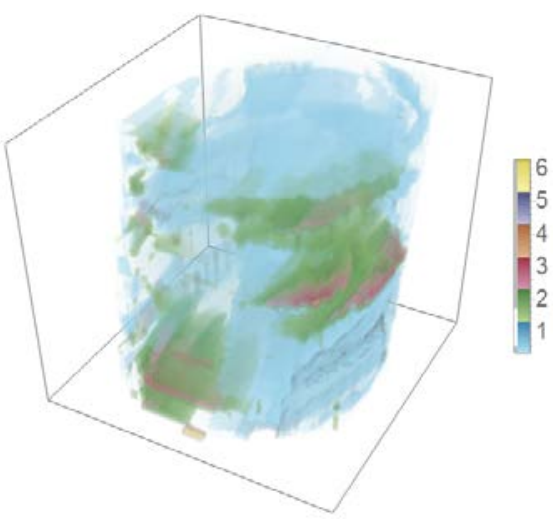

b

Fig. 3. Results of calculation: (a) - distribution of eigenvectors (red - the $1^{\text {st }}$ eigenvector, green - the $2^{\text {nd }}$ eigenvector, blue - the $3^{\text {rd }}$ eigenvector), (b) - distribution of fractional anisotropy.

In this case, average fabric tensor and its standard deviation can be reconstructed: 


$$
\langle H\rangle=\left(\begin{array}{ccc}
0.320 & 1 \cdot 10^{-4} & -2 \cdot 10^{-4} \\
1 \cdot 10^{-4} & 0.322 & 1 \cdot 10^{-4} \\
-2 \cdot 10^{-4} & 1 \cdot 10^{-4} & 0.320
\end{array}\right) \pm\left(\begin{array}{lll}
3 & 2 & 2 \\
2 & 3 & 2 \\
2 & 2 & 4
\end{array}\right) \cdot 10^{-3}
$$

\section{Conclusion}

The technique of direct fabric tensor calculation according to computed tomography data is presented. Additionally, a method and algorithm for analyzing bone objects is considered. Fractional anisotropy was used to describe the degree of anisotropy. The described procedure was used to analyze the micro-computed tomography data of a bone sample. The specimen was meshed with 280 elements and the fabric tensor was reconstructed for each element. Eigenvectors, eigenvalues and fractional anisotropy were calculated. The MIL distribution was analyzed using the described method. It was shown that the fabric tensor is constant throughout the bone sample and the degree of anisotropy is small.

The work was supported by the Russian Foundation for Basic Research and Government of Republic of Tatarstan within the scientific projects № 18-41-160025 and № 20-01-00535.

\section{References}

1. O. Sachenkov, R. Hasanov, P. Andreev, Y. Konoplev, Determination of muscle effort at the proximal femur rotation osteotomy, IOP Conf. Ser. Mater. Sci. Eng., 158(1), 012079 (2016).

2. O.A. Sachenkov, R.F. Hasanov, P.S. Andreev, Yu.G. Konoplev, Numerical study of stress-strain state of pelvis at the proximal femur rotation osteotomy, Russ. J. Biomech., 20(3), 220-232 (2016), doi: 10.15593/RJBiomech/2016.3.06.

3. D. Kaplun, M. Golovin, A. Sufelfa, O. Sachenkov, K. Shcherbina, V. Yankovskiy, E. Skrebenkov, O.A. Markelov, M.I. Bogachev, Three-dimensional (3D) model-based lower limb stump automatic orientation, Appl. Sci., 10(9), 3253 (2020), doi: 10.3390/app10093253.

4. R. Pryazhevskiy, I. Akhtyamov, A. Morgunova, H.M. Jihad, A. Nevzorov, O. Sachenkov, Modeling of contact interaction of an endoprosthetic knee joint, Adv. Intell. Syst. Comput., 1018, 612-617 (2020), doi: 10.1007/978-3-030-25629-6_95.

5. R.A. Kayumov, I.Z. Muhamedova, B.F. Tazyukov, F.R. Shakirzjanov, Parameter determination of hereditary models of deformation of composite materials based on identification method, J. Phys. Conf. Ser., 973(1), 012006 (2018), doi: 10.1088/17426596/973/1/012006.

6. R.A. Kayumov, Structure of nonlinear elastic relationships for the highly anisotropic layer of a nonthin shell, Mech. Compos. Mater., 35(5), 409-418 (1999), doi: 10.1007/BF02329327.

7. D.V. Berezhnoi, E.V. Antonova, I.S. Balafendieva, N.R. Vildanova, V.V. Miheev, L.R. Sekaeva, Investigation of nonlinear deformation of solid and porous elements of three-dimensional structures, J. Phys. Conf. Ser., 1158(2), 022023 (2019), doi: 10.1088/1742-6596/1158/2/022023.

8. D.V. Berezhnoi, N.F. Gabsalikova, V.G. Izotov, V.V. Miheev, Modeling of deformation of ground media on the basis of the particle method in a two-dimensional formulation, IOP Conf. Ser. Mater. Sci. Eng., 208(1), 012028 (2017), doi: 10.1088/1757-899X/208/1/012028.

9. S. Gupta, P. Dan, Bone geometry and mechanical properties of the human scapula 
using computed tomography data, Trends Biomater. Artif. Organs, 17(2), 61-70 (2004).

10. T.S. Kaneko, M.R. Pejcic, J. Tehranzadeh, J.H. Keyak, Relationships between material properties and CT scan data of cortical bone with and without metastatic lesions, Med. Eng. Phys., 25(6), 445-454 (2003).

11. F. Eggermont, L.C. Derikx, J. Free, R. van Leeuwen, Y.M. van der Linden, N. Verdonschot, E. Tanck, Effect of different CT scanners and settings on femoral failure loads calculated by finite element models, J. Orthop. Res., 36(8), 2288-2295 (2018).

12. J.Y. Rho, M.C. Hobatho, R.B. Ashman, Relations of mechanical properties to density and CT numbers in human bone, Med. Eng. Phys., 17(5), 347-355 (1995).

13. N. Kharin, O. Vorobyev, P. Bolshakov, O. Sachenkov, Determination of the orthotropic parameters of a representative sample by computed tomography, J. Phys. Conf. Ser., 1158(3), 032012 (2019).

14. O. Gerasimov, N. Kharin, O. Vorobyev, E. Semenova, O. Sachenkov, Determination of the mechanical properties distribution of the sample by tomography data, J. Phys. Conf. Ser., 1158(2), 022046 (2019).

15. N.V. Kharin, O.V. Vorobyev, D.V. Berezhnoi, O.A. Sachenkov, Construction of a representative model based on computed tomography, PNRPU Mech. Bull., 3, 95102 (2018).

16. O.A. Sachenkov, O.V. Gerasimov, Y.V. Koroleva, D.A. Mukhin, V.V. Yaikova, I.F. Akhtyamov, F.V. Shakirova, D.A. Korobeynikova, K.K. Chzhi, Building the inhomogeneous finite element model by the data of computed tomography, Russ. J. Biomech., 22(3), 291-303 (2018).

17. P. Marcián, J. Wolff, L. Horáčková, J. Kaiser, T. Zikmund, L. Borák, Micro finite element analysis of dental implants under different loading conditions, Comput. Biol. Med., 96, 157-165 (2018).

18. L. Giovannelli, J.J. Rodenas, J.M. Navarro-Jimenez, M. Tur, Direct medical imagebased Finite Element modelling for patient-specific simulation of future implants, Finite Elem. Anal. Des., 136, 37-57 (2017).

19. T.P. Harrigan, R.W. Mann, Characterization of microstructural anisotropy in orthotropic materials using a second rank tensor, J. Mater. Sci., 19, 761-767 (1984).

20. T.A. Carniel, B. Klahr, E.A. Fancello, On multiscale boundary conditions in the computational homogenization of an RVE of tendon fascicles, J. Mech. Behav. Biomed. Mater., 91, 131-138 (2019).

21. P. Marcián, Z. Florian, L. Horáčková, J. Kaiser, L. Borák, Microstructural finiteelement analysis of influence of bone density and histomorphometric parameters on mechanical behavior of mandibular cancellous bone structure, SSP, 258, 362-365 (2017).

22. F. Eggermont, L.C. Derikx, N. Verdonschot, I.C.M. Van Der Geest, M.A.A. De Jong, A. Snyers, Y.M. Van Der Linden, E. Tanck, Can patient-specific finite element models better predict fractures in metastatic bone disease than experienced clinicians, Bone Joint Res., 7(6), 430-439 (2018).

23. G. Hettich, R.A. Schierjott, H. Ramm, H. Graichen, V. Jansson, M. Rudert, F. Traina, T.M. Grupp, Method for quantitative assessment of acetabular bone defects, J. Orthop. Res., (2018), doi: 10.1002/jor.24165.

24. F. Marwa, E. Y. Wajih, L. Philippe, M. Mohsen, Improved USCT of Paired Bones Using Wavelet-based Image Processing, IJIGSP, 10(9), 1-9 (2018), doi: 10.5815/ijigsp.2018.09.01.

25. K.P.K. Mithun, A. Gauhar, M.R. Mohammad, H. A.S.M. Delowar, Automatically 
Gradient Threshold Estimation of Anisotropic Diffusion for Meyer's Watershed Algorithm Based Optimal Segmentation, IJIGSP, 6(12), 26-31 (2014), doi: 10.5815/ijigsp.2014.12.04.

26. K.P.K. Mithun, M.R. Mohammad, Metal Artifact Reduction from Computed Tomography (CT) Images using Directional Restoration Filter, IJITCS, 6(6), 47-54 (2014), doi: 10.5815/ijitcs.2014.06.07.

27. O. Gerasimov, F. Shigapova, Y. Konoplev, O. Sachenkov, Evaluation of the stressstrain state of a one-dimensional heterogeneous porous structure, IOP Conf. Ser. Mater. Sci. Eng., 158(1), 012036 (2016).

28. O. Gerasimov, F. Shigapova, Y. Konoplev, O. Sachenkov, The evolution of the bone in the half-plane under the influence of external pressure, IOP Conf. Ser. Mater. Sci. Eng., 158(1), 012037 (2016).

29. E. Semenova, O. Gerasimov, E. Koroleva, N. Ahmetov, T. Baltina, O. Sachenkov, Automatic processing and analysis of the quality healing of derma injury, Adv. Intell. Syst. Comput., 831, 107-113 (2019).

30. N. Chernov, H. Ma, Computer Vision, Least squares fitting of quadratic curves and surfaces (Book chapter), 287-302 (2011).

31. V.V. Yaikova, O.V. Gerasimov, A.O. Fedyanin, M.A. Zaytsev, M.E. Baltin, T.V. Baltina, O.A. Sachenkov, Automation of bone tissue histology, Front. Phys., 7, 91 (2019), doi: 10.3389/fphy.2019.00091.

32. A. Ridwan-Pramana, P. Marcian, L. Borak, N. Narra, T. Forouzanfar, J. Wolff, Finite element analysis of 6 large PMMA skull reconstructions: A multi-criteria evaluation approach, PLoS ONE, 12, e0179325 (2017), doi: 10.1371/journal.pone.0179325. 